\title{
Multifocal Polyostotic Aneurysmal Bone Cyst of Pelvic Bones
}

\author{
Salem Bouomrani ${ }^{1,2 *}$, Sihem Riahi ${ }^{1}$ \\ ${ }^{1}$ Department of Internal medicine, Military Hospital of Gabes, Tunisia \\ ${ }^{2}$ Department of Internal medicine, Sfax Faculty of Medicine, University of Sfax. Sfax 3029. Tunisia
}

*Corresponding author: Salem Bouomrani, Department of Internal medicine, Military Hospital of Gabes. Gabes 6000. Tunisia.

Received Date: July 06, 2020

Published Date: July 24, 2020

\begin{abstract}
Aneurysmal bone cyst $(\mathrm{ABC}$ ) is a very rare benign osteolytic bone tumor representing less than $2 \%$ of all bone tumors and 5 to $6 \%$ of benign bone tumors. Atypical or unusual variants of ABC group together unusual clinical presentations, unusual complication, rare locations, and unusual age of occurrence, representing a real diagnostic challenge for clinicians. From all so-called unusual variants of ABC, multifocal one is particularly exceptional with only seven cases described metachronously in the world literature. We report the original case of a bifocal synchronous poly-ostotic and locally aggressive $\mathrm{ABC}$ involving the right ilium and the left superior pubic ramus in 19-year-old Tunisian man.
\end{abstract}

Keywords: Aneurysmal bone cyst; Ilium; Pubis; Poly-ostotic; Multifocal aneurysmal; Bone cyst

\section{Introduction}

Aneurysmal bone cyst $(\mathrm{ABC})$ was described for the first time by Jaffe and Lichtenstein in 1942 as a particular variant of solitary unicameral bone cyst [1]. It is currently defined by the World Health Organization's histologic classification of bone tumors as "expansive and osteolytic benign bone tumor consisting of bloodfilled cavities lined by connective tissue septa" [2]. ABC is very rare representing only $1-2 \%$ of all bone tumors and 5-6\% of benign bone tumors [3]. This tumor can occur at any age but remains particularly frequent in children and adolescents with a maximum incidence before the age of 20 years [3,4]. Likewise, all bones can be affected by this tumor; the long bones are the most frequently involved [3,4]. Classically this bone pseudotumor is unique [2-4] but can exceptionally be multiple [5], recurrent [6], or with distant metatstases [7] posing a real diagnostic challenge for clinicians [5-7]. From the so-called "atypical" or "unusual" variants of $A B C$, multifocal presentation is exceptional with only few sporadic cases reported in the medical literature $[5,6,8]$. We report the original case of a bifocal, synchronous, and locally aggressive $\mathrm{ABC}$ in a 19-year-old Tunisian man.

\section{Case report}

A 19-year-old patient, without pathological medical history, consulted our department for pain and progressive swelling in the fold of the left groin, worsening progressively over the past two months.

The somatic examination noted left limping, pain in mobilization of the left leg and hip, and a mass in the fold of the left groin. This swelling was $5 \mathrm{~cm}$ long, solid, and sensitive. No other anomalies were noted. Basic biological tests were within normal limits, particularly total blood count, erythrocyte sedimentation rate, C-reactive protein, serum calcium, phosphoremia, alkaline phosphatase, lactate dehydrogenase, creatinine, muscle enzymes, and electrophoresis of serum proteins. 
Plain radiographs of the pelvis demonstrated important osteolysis with complete disappearance of the left superior pubic ramus (Figure 1). Soft tissue ultrasound demonstrated heterogeneous, multiloculated, and predominantly fluid mass in the fold of the left groin (Figure 2). Pelvic CT scan objectified a voluminous, poorly limited, and aggressive expansive process destroying the whole of the left superior pubic ramus and invading the soft parts of the left groin (Figure 3). This tumor was associated to a second osteolytic, blowing, and multi-septate lesion of the posterior part of the right iliac wing which was not visible on standard radiography (Figure 4 ).

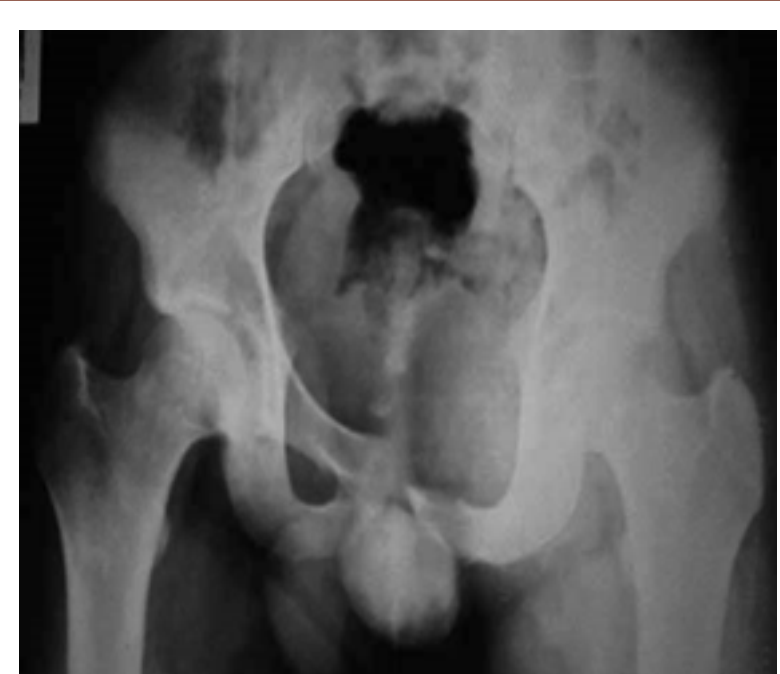

Figure1: Anteroposterior view of X-ray of the pelvis: complete disappearance of the left superior pubic ramus.

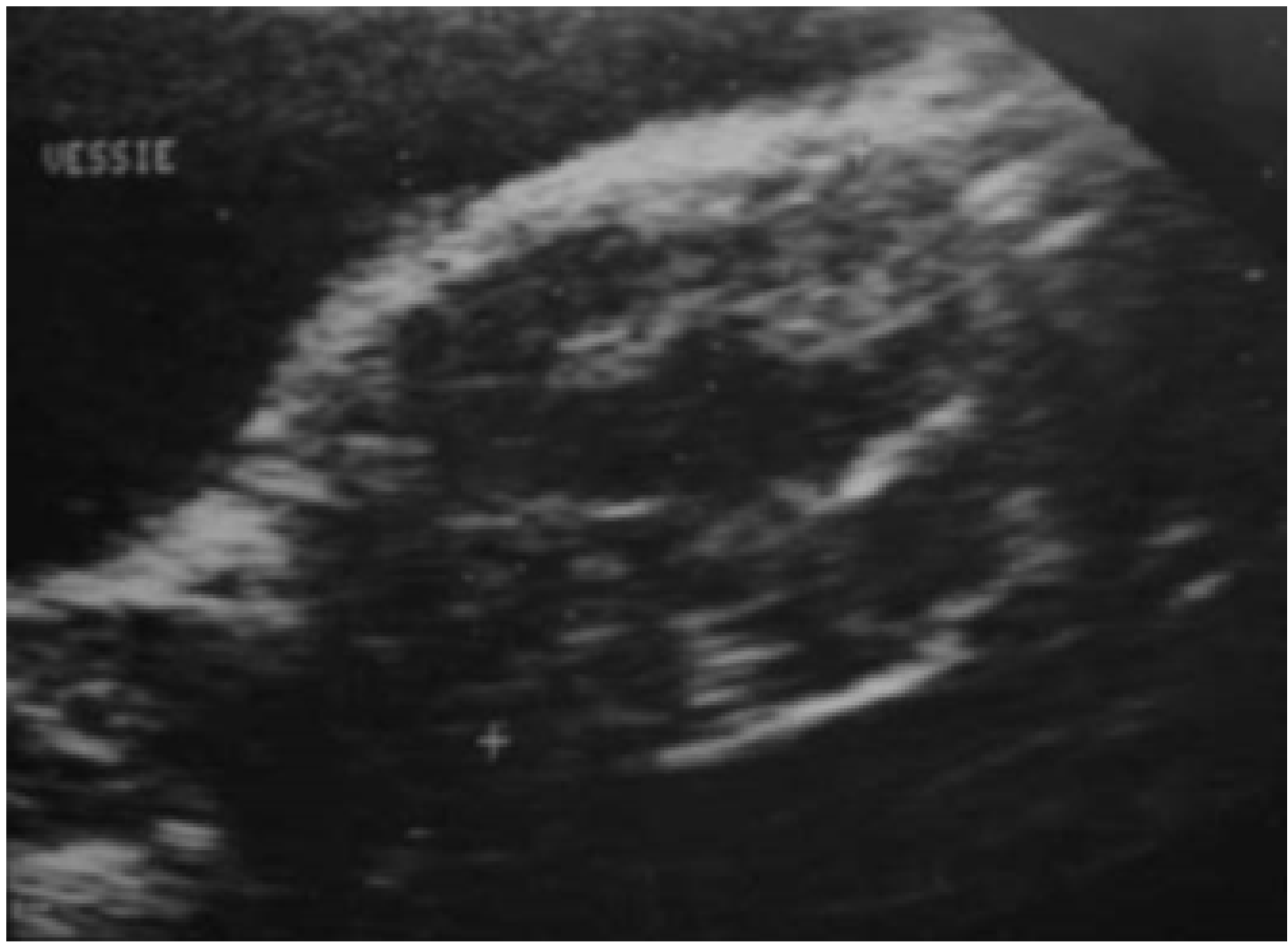

Figure2: Soft tissue ultrasound: heterogeneous, multi-partitioned, and predominantly fluid mass in the fold of the left groin. 


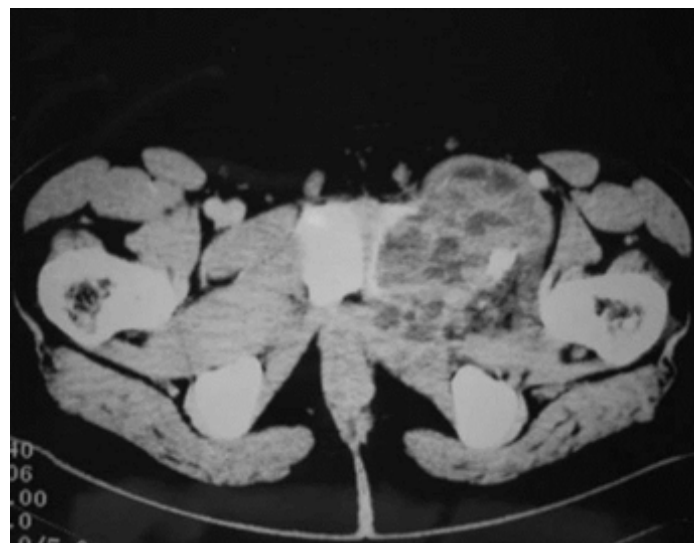

Figure3: Axial pelvic CT without injection: voluminous and aggressive expansive process destroying the whole of the left superior pubic ramus.

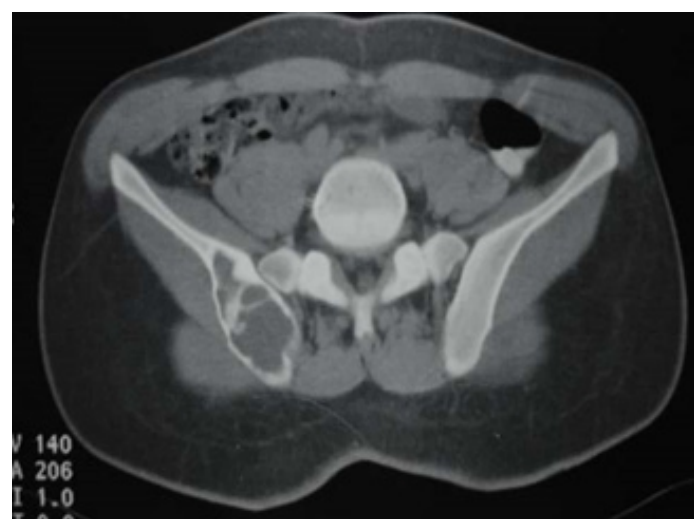

Figure4: Axial pelvic CT without injection: osteolytic, blowing, multi-septate lesion of the posterior part of the right iliac wing (second location)

The patient was operated with the preoperative diagnosis of Ewing sacroma. He had a total excision of the two tumors with reconstruction of the acetabulum. The anatomopathological study concluded with an $\mathrm{ABC}$ with double localization (ilium and pubis). The post-operative follow-ups were simple with a good subsequent clinical and radiological outcome.

\section{Discussion}

Atypical and unusual variants of $\mathrm{ABC}$ group together unusual clinical presentations (solid $\mathrm{ABC}$, bifocal $\mathrm{ABC}$, multiple $\mathrm{ABC}$, and extraosseous or soft tissue $\mathrm{ABC}$ ), unusual complications (pathological fractures), rare locations, and the occurrence at an unusual age [9-11]. These forms are rare and represent a real diagnostic challenge for clinicians [9-11]. Multifocal variant of $\mathrm{ABC}$ is particularly exceptional, and since its initial description by Sundaram M et al in 1997 [12], only a few sporadic cases have been reported in the world literature [5,6,8,13-15]. Indeed, the systemic review of English literature made in 2020 by Angelini A et al, found only seven cases of multifocal $\mathrm{ABC}$ [8]. There are two types of multifocal $\mathrm{ABC}$ : mono-ostotic multifocal $\mathrm{ABC}$ when multiple cystic tumors occur in the same bone and poly-ostotic multifocal $\mathrm{ABC}$ when multiple lesions occur in multiple bones $[8,15]$.

This variant seems to be particularly frequent in male patients (5 patients/7 in Angelini A et al's review), and the most reported sites are the femur and the tibia [8]. No case of multifocal $A B C$ of pelvic bones was found.The multifocal forms of $\mathrm{ABC}$ are in the majority of cases described metachronously [5,6,8,13-15], the synchronous occurrence in the same patient is exceptional. Only one case of two separate synchronous $\mathrm{ABC}$ of the humerus was found in Mohaidat ZM et al series [9]. These multifocal and locally aggressive forms pose the problem of differential diagnosis with malignant bone tumors (cancers and metastases), particularly that the malignant transformation of ABC into osteosarcoma has been reported [7]. The standard treatment for these tumors is surgery $[5,6,11,12]$. Recently, biotherapy with Denosumab seems to be promising for the multifocal and recurrent forms of $\mathrm{ABC}$ [16]. Our observation is distinguished by the synchronous occurrence and the unusual localization in the pelvic bones (ilium and superior pubic ramus).

\section{Conclusion}

Multifocal aneurysmal bone cyst is an exceptional unusual variant of this osteolytic benign bone tumor with only seven sporadic cases previously reported in the literature. Our observation is distinguished by the synchronous occurrence, the poly-ostotic involvement, and the unusual localization in the pelvic bones (ilium and pubis). As rare as it is, this atypical clinical presentation of $A B C$ deserves to be known by health practitioners. 


\section{Acknowledgement}

None.

\section{Conflicts of Interest}

None.

\section{References}

1. Jaffe HL, Lichtenstein L (1942) Solitary unicameral bone cyst: With emphasis on the roentgen picture, the pathologic appearance and the pathogenesis. Arch Surg 44: 1004-1025.

2. Schajowicz F, Sissons HA, Sobin LH (1995) The World Health Organization's histologic classification of bone tumors. A commentary on the second edition. Cancer 75(5): 1208-1214.

3. Puri A, Hegde P, Gulia A, Parikh M (2020) Primary aneurysmal bone cysts. Bone Joint J 102-B(2):186-190.

4. Mascard E, Gomez-Brouchet A, Lambot K (2015) Bone cysts: unicameral and aneurysmal bone cyst. Orthop Traumatol Surg Res 101(1 Suppl): S119-S127.

5. Scheil-Bertram S, Hartwig E, Bruderlein S, Melzner I, Von Baer A, et al. (2004) Metachronous and multiple aneurysmal bone cysts: a rare variant of primary aneurysmal bone cysts. Virchows Arch 444(3): 293299.

6. Karkuzhali P, Bhattacharyya M, Sumitha P (2007) Multiple soft tissue aneurysmal cysts: An occurrence after resection of primary aneurysmal bone cyst of fibula. Indian J Orthop 41(3): 246-249.
7. Van de Luijtgaarden AC, Veth RP, Slootweg PJ, Wijers-Koster PM, Schultze Kool LJ, et al. (2009) Metastatic potential of an aneurysmal bone cyst. Virchows Arch 455(5): 455-459.

8. Angelini A, Mavrogenis AF, Pagliarini E, Igoumenou VG, Gulia A, et al. (2020) Rare aneurysmal bone cysts: multifocal, extraosseous, and surface variants. Eur J Orthop Surg Traumatol 30(6): 969-978.

9. Mohaidat ZM, Al-Gharaibeh SR, Aljararhih ON, Nusairat MT, Al-Omari AA (2019) Challenges in the Diagnosis and Treatment of Aneurysmal Bone Cyst in Patients with Unusual Features. Adv Orthop 2019: 2905671.

10. Bouomrani S, Ben Ayed M, Trabelsi S, Belgacem N (2018) Extraosseous Aneurysmal Bone Cyst of the Ankle. ARC Journal of Orthopedics 3(2): 14-16.

11. Bouomrani S, Mesfar R, Ben Ayed M, Guermazi M, Belgacem N (2019) Pathologic fracture revealing solid aneurysmal bone cyst of the distal radius. Journal of Orthopaedics Trauma Surgery and Related Research 14(2): 34-36

12. Sundaram M, McDonald DJ, Steigman CK, Bocchini T (1997) Metachronous multiple aneurysmal bone cysts. Skelet Radiol 26(9): 564-567.

13. Amer HZ, Mayerson JL, Klein MJ, Baker PB (2012) Metachronous aneurysmal bone cyst in a 9-year-old boy: case report and review of the literature. J Pediatr Orthop B 21(2): 187-191.

14. Donigan JA, Kebaish KM, McCarthy EF (2003) Metachronous aneurysmal bone cysts with involvement of the humerus and the thoracic vertebrae. Skeletal Radiol 32(8):468-471.

15. Naylor WM, Collier CD, Redline RW, Getty PJ (2019) Metachronous, 